\title{
De reclusiones maternas y habitantes de los «no lugares». La gestión del acompañamiento paterno en el marco de los partos hospitalarios públicos.
}

\author{
José Manuel Hernández Garre, Paloma Echevarría Pérez y María José \\ Gomariz Sandoval \\ Universidad Católica de Murcia ${ }^{1}$
}

DOI: http://dx.doi.org/10.5565/rev/periferia.518

\section{Resumen}

La evidencia científica muestra como el apoyo emocional continuo dado por el padre durante el parto reduce la duración del mismo, la necesidad de analgesia y aumenta las probabilidades de parto vaginal espontáneo, tratándose además de un derecho para las madres que avalan la legislación vigente. Partiendo de estas premisas el artículo explora las experiencias maternas y paternas ligadas al acompañamiento en el marco de los partos que transcurren en los hospitales públicos. La información se obtuvo a través de la realización de una serie de entrevistas semiestructuradas realizadas a madres y padres que habían sido atendidos en un centro sanitario público. Los testimonios hablan de unos rituales en paritorio que recluyen a las madres y excluyen a los padres, desplazando el protagonismo a los asistentes. La conclusión es que se trata de un itinerario asistencial cargado de ideologías productivistas y biopolíticas que da prelación a las rutinas biológicas sobre las necesidades de acompañamiento de las madres y los padres.

Palabras clave: Parto hospitalario público; apoyo emocional; acompañamiento paterno.

\begin{abstract}
Scientific evidence shows how the continuous emotional support given by the father during labor reduces the duration of labor, the need for analgesia and increases the likelihood of spontaneous vaginal delivery, besides being a right for mothers who supports the legislation. Based on these assumptions the article explores maternal and paternal experiences related to accompaniment under the public hospital births. The information was obtained through conducting a series of semistructured interviews with parents who had been treated at a public health center. The testimonies speak of rituals that recluse mothers and exclude parents, moving the stage to the assistants. The conclusion is that it is a care pathway loaded of productivist and bio-political ideologies that gives priority to biological routines on the needs of accompanying of mothers and fathers.
\end{abstract}

\footnotetext{
${ }^{1}$ Enviar correspondencia a: José Manuel Hernández Garre (jmhernandez@ucam.edu)
} 
Keywords: Public hospital birth; interventionist birth; emotional support; paternal accompanying.

\section{Introducción}

El parto es una experiencia umbral, casi liminar, una especie de rito de paso (Van Gennep 1986) situado en los terrenos fronterizos de la capacidad física y moral de las madres. Estamos ante un trance en el que se ponen a prueba los recursos de afrontamiento psicológicos más íntimos, por lo que el aspecto emocional del proceso juega un papel crucial. Se trata de una realidad que la evidencia científica ha cuantificado en términos de resultados obstétricos, abundando la literatura que apunta que el apoyo emocional continuo, dado preferentemente por un familiar significativo para la madre como el padre $^{2}$, se traduce en una menor sensación dolorosa, en un menor uso de la analgesia epidural, en un mayor índice de partos vaginales normales y en un mayor grado de satisfacción general de la madre (Lally et al. 2008; Hodnett et al. 2012; 2013). Además el acompañamiento en el parto hace el trabajo de parto más breve, disminuyendo las instrumentaciones y cesáreas y mejorando la adaptación neonatal al medio extrauterino (Klaus y Kennel 1993; Hofmeryr et al. 1991; Hemminki, Virta y Koponen 1990; Enkin, Keirse y Renfrew 1989). Las hipótesis apuntan a que el apoyo mejora la sensación de control y de competencia de la madre, reduciendo la dependencia a las intervenciones médicas y el uso de la epidural, que puede enlentecer el expulsivo del bebé. Todo ello redundaría en una disminución del nivel de estrés mejorando el paso del feto a través de una pelvis materna más relajada.

Todas estas evidencias científicas han llevado a la Organización Mundial de la Salud (OMS) a emitir diferentes documentos (OMS 1985; 1996) en favor de una mayor humanización de los partos hospitalarios que promocione la consecución de partos normales, promocionando una mejor experiencia materna que incluya el apoyo

\footnotetext{
${ }^{2}$ En este sentido hay que explicitar que el padre no es la única figura que puede dar apoyo emocional durante el parto, existiendo también otras figuras de acompañamiento que también pueden aportar grandes satisfacciones a las madres durante el proceso como son las «doulas» u otras mujeres de confianza. También hay que apuntar que existe un debate abierto sobre la pertinencia de la presencia del padre en el parto, ya que desde algunas corrientes se apunta a que en algunos casos podrían incluso entorpecer el proceso, sobre todo en el caso de parejas poco compenetradas. Lógicamente no son este tipo de situaciones las que se recogen en el presente estudio.
} 
emocional efectivo. Recomendaciones que han sido también secundadas en España por diferentes organismos como Ministerio de Sanidad y Consumo (MSC 2010a; 2010b; 2007), la Sociedad Española de Ginecología y Obstetricia (SEGO 2008) o la Federación de Asociaciones de Matronas de España (FAME 2008), que insisten en la necesidad de promocionar un entorno humanizado que satisfaga las necesidades emocionales de las madres y facilite los procesos de acompañamiento durante el parto. Acompañamiento que también viene expresamente recogido en la legislación vigente, concretamente en el punto 3 del artículo 22 del Título III, Capítulo I, de la Ley 3/2009 de Derechos y Deberes de los Usuarios, que hace mención expresa al derecho a las madres a estar acompañadas por personas significativas ajenas a las instituciones sanitarias durante su proceso de parto.

El papel del acompañamiento paterno durante el parto también está bien definido por sociedades científicas como el American College of Nurse-Midwive que en documento sobre "La gestión del dolor durante el parto" (2013) le otorga funciones muy variopintas. Entre ellas se encuentra el ayudar a la madre a encontrar y mantener el ritmo de las respiraciones o a cronometrar el intervalo de las contracciones durante la dilatación, el darle masajes en la espalda o tomarle la mano en silencio, el ofrecerle hielo, agua o zumo, el ayudarla a cambiar de posición o a sostenerla, el bajar la luz o poner música suave, el pone paños tibios en su espalda, el animarla durante la contracción para ayudarle a llevar el dolor, el entretenerla para disminuir la sensación álgida, el tranquilizarla y calmarla en los momentos de mayor tensión, el estar atento a sus necesidades (sed, frío, calor, etc.) o el solicitar atención del especialista si la madre lo requiere. El papel del padre es pues de soporte en un proceso en el que lógicamente los auténticos protagonistas son las madres y sus bebés, papel no simétrico en el paritorio que es de sentido común y no implica menos igualdad entre los progenitores.

Estamos pues ante un debate muy actual que versa sobre la humanización de los procesos asistenciales hospitalarios, de un tema de especial sensibilidad al tratarse de cómo se atiende el principio de la vida. En este «habitus» se enmarca el presente estudio cuyo objetivo ha sido explorar desde una perspectiva fenomenológica las experiencias maternas y paternas ligadas a los procesos de acompañamiento en el marco de los partos hospitalarios públicos. El análisis se ha realizado desde un posicionamiento teórico crítico que desde la perspectiva 
personalista ${ }^{3}$ y de género ${ }^{4}$ reflexiona sobre el respecto y humanización de los itinerarios clínicos ligados a la maternidad.

\section{Metodología}

El estudio se ha realizado desde un enfoque fenomenológico que pretende aproximarse a los elementos explicativos clave de los aspectos estructurales de la gestión del acompañamiento en los partos de los hospitales públicos (Bourdieu y Wacquant 1995). Se trata de una perspectiva cada vez más utilizado en el marco de los estudios sanitarios, ya que enriquece sobremanera los datos estadísticos, típicos de las investigaciones puramente cuantitativas, incorporando conocimiento de profundidad sobre los porqués y los aspectos vivenciales de las realidades clínicas (Caprara y Lamdin 2008; Janes, Stall y Gifford 1986). La perspectiva fenomenológica posibilita, además, enmarcar procesos como el que nos ocupa en un contexto social y moral particular, facilitando, así, la interpretación del sentido de las prácticas que se dan en él (Hernández Garre, 2013; Kleinman y Benson 2004; Kleinman 1988). A ello se suma la conveniencia de incorporar el punto de vista «emic» de las propias madres y sus acompañantes, partiendo de la base de que ellos tienen su propio punto de vista sobre la gestión del acompañamiento en los hospitales públicos (Taylor y Bogdan 1987).

El estudio se inscribió en el marco de una tesis doctoral del Área de Antropología Social de la Facultad de Filosofía de la Universidad de Murcia. El contexto de la investigación fue el paritorio y área de maternidad de un hospital público del sur de España que da cobertura a 158.680 habitantes, presentando una media de 1705 partos anuales (4.67 al día) con cifras medias elevadas tanto de cesáreas ( $31 \%$ ) como de partos instrumentados (25\%), inducciones de parto (29\%), episiotomías (50\%), uso de oxitocina (75\%), amniorrexis artificiales (50\%) y administración de analgesia locoregional (60\%). La política del hospital, en consonancia con la Ley 3/2009 de Derechos y Deberes de los Usurarios, recogía expresamente el derecho

3 El personalismo es una corriente filosófica representada por autores como Kant, Kierkegaard, Maritain, Mounier, Marcel o Wojtyla que pone el énfasis en la persona. Considera al hombre como un ser relacional, esencialmente social y comunitario, un ser libre, trascendente y con un valor en sí mismo que le impide convertirse en un objeto como tal.

${ }^{4}$ Estamos ante un movimiento ideológico iniciado por autores como Margaret Mead, Simone Beavouir o John Money, que reflexiona sobre todas las representaciones culturales y sociales que construyen la identidad masculina y femenina. El objetivo es examinar el impacto del sexo en las oportunidades de las personas, sus roles sociales y las interacciones mutuas. 
de las madres a estar acompañadas por un familiar durante el proceso de parto. Por motivos de confidencialidad, y siguiendo las indicaciones del centro, se ha preferido mantener el anonimato del mismo.

La técnica de recogida de datos fue la entrevista semiestructurada realizada, de forma individual y mediante un guión abierto, a mujeres y acompañantes que habían sido asistidos en el centro hospitalario. Durante su realización se pretendió generar una línea discursiva no fragmentada o precodificada que no coartar los testimonios y enriqueciera el discurso desde el propio punto de vista de los actores del proceso (Grele 1991). El lugar de realización fue la sala de lactancia de la planta de maternidad de dicho hospital, espacio privado y sin interferencias, y el periodo entre el 1 de Febrero y el 31 de Marzo del año 2010. Éstas se realizaron tras conseguir la autorización del Centro y el consentimiento informado de los participantes, y siempre durante los primeros días postparto, antes del alta hospitalaria, con el objetivo de que el relato no fuera tamizado por el tiempo. En todo momento se respetó la confidencialidad de los participantes mediante la asignación de un código que no permite su identificación. El tamaño final de la muestra fue de 20 participantes (15 madres y 5 padres), número que se alcanzó tras seguir el criterio de saturación, entendiendo éste como la situación a la que se llega cuando los testimonios empiezan a ser repetitivos y se considera que los nuevos testimonios no aportan ya datos significativos (Bertaux y Bertaux 1993). La preeminencia de madres en la muestra fue consecuencia de la dificultad de acceder a los testimonios de los padres, que en muchos casos no se encontraban presentes en el postparto hospitalario por razones laborales o de otra índole, estando en muchas ocasiones las madres acompañadas por las abuelas, tías u otros familiares de los bebés. Dificultad que se ha compensado dándoles peso en los testimonios en consonancia con el fin del estudio que pretendía recoger sus experiencias de acompañamiento.

Con el objetivo de mejorar la validez interna de la muestra se siguió el criterio de intersubjetividad y el ya comentado criterio de saturación del discurso. Debido a la heterogeneidad de los perfiles de los posibles participantes en el estudio, la multitud de factores vivenciales y biográficos que podían influir en la percepción de los procesos de acompañamiento en el parto, se decidió homogeneizar la muestra en base a los siguientes criterios de selección: edad materna entre 25 y 35 años ( $74 \%$ de la población), nacionalidad española (67\% de la población), situación de 
pareja fija y heterosexual, nivel socioeconómico estable, primiparidad ( $54 \%$ de la población), embarazo de bajo riesgo ( $76 \%$ de la población) y gestación deseada ( $91 \%$ de la población $)^{5}$. Con ello se pretendió recoger los perfiles más prevalentes de la población de estudio y eliminar factores de confusión como las edades extremas (SEGO 2008), las diferentes perspectivas culturales (Luque y Oliver 2005), las relaciones inestables (Schwarz 2008), las dificultades económicas (SEGO 2008), las experiencias previas negativas (Baroni, Vilela, Scorsolini 2013) o las patologías maternas o embarazos no deseados (SEGO 2008), que según la evidencia pueden distorsionar las vivencias influyendo negativamente en la percepción del mismo e introducción de factores de confusión ajenos a la naturaleza del estudio. De la misma manera se evitaban también situaciones maternas o fetales que aconsejaran un control ferro del proceso dificultando el acompañamiento como consecuencia de situaciones clínicas patológicas.

Los relatos fueron grabados digitalmente e íntegramente transcritos introduciendo para su análisis un orden en el discurso que permitiera recuperar los datos en base a las categorías de análisis (Coffey y Atkinson 2005, Ibañez 1979). Éstas se obtuvieron tras la revisión de la bibliografía y la aproximación a las primeras entrevistas piloto, fijando tres categorías generales: la gestión del acompañamiento por parte del personal sanitario, el papel asignado a madres y padres durante el parto desde la perspectiva de género, y el valor simbólico que las madres y los padres les dan al mismo. Para la selección de los fragmentos del discurso en base a las categorías de análisis se utilizó el programa de gestión de datos cualitativos MAX.QDA. Los testimonios fueron bastante homogéneos para las tres categorías de análisis, reflejando pese a las discrepancias las principales vivencias ligadas al acompañamiento en los partos. El análisis se realizó como se ha comentado desde una perspectiva crítica personalista y de género en defensa de la humanización de los procesos clínicos, de la dignidad ontológica de las madres y sus acompañantes y de la asunción de roles sexuales igualitarios.

Los resultados que aquí se muestran no pretenden ser categóricos ni extrapolables a todas las madres y sus acompañantes, sino reflejar ciertas circunstancias clínicas

\footnotetext{
${ }^{5}$ Las cifras hacen referencia a los porcentajes recogidos en los registros del centro, tomando como población el total de mujeres que dieron a luz en el año 2010. No existen registros sobre el tipo de pareja y el nivel socieconómico por lo que el acceso a la información se consiguió mediante un sondeo previo a los participantes del estudio.
} 
que se dan en los centros hospitalarios públicos en torno al parto y los procesos de acompañamiento. En este sentido, las conclusiones solo son extrapolables al perfil de madres seleccionadas para el estudio, y están además acotadas por las típicas limitaciones referidas a la neutralidad y simetría de las interpretaciones (Romaní 1992), y por la dificultad de hacer extrapolaciones generales partiendo de informadores tipo (Mauss 1966), dificultades propias de las investigaciones de carácter fenomenológico.

\section{Resultados/Discusión}

\section{De reclusiones rituales $y$ exclusiones paternas. La gestión del acompañamiento paterno en los partos hospitalarios}

Del análisis de los argumentos maternos deviene la reflexión de que los procesos de acompañamiento durante el nacimiento, y por tanto las experiencias que se derivan de ellos, están determinados por la propia dinámica asistencial hospitalaria. Dinámica que en función de los alegatos parece dar prelación a la intervención técnica sobre los «organismos grávidos», propiciando una rigidez estructural que lleva, en muchas ocasiones, a la clausura de las madres y a la invisibilidad de los padres. Los relatos hablan, de esta manera, de un ceremonial litúrgico que parece precisar, para investirse de atribuciones hieráticas, del encierro de las parturientas, retiro que marca la sacralidad de lo que allí está aconteciendo.

Como diría Menéndez, parece que estamos ante una tendencia en la que cada vez se excluye "más la palabra de <las gestantes> y ello sobre todo por factores de carácter institucional" (2000: 179). Circunstancia que podría estar favoreciendo el aislamiento materno a lo largo del itinerario de los partos hospitalarios: «YO supliqué mucho arriba que subiera, porque yo sola no quería estar» ( $\mathrm{x} x \Psi)$. La razón de este aislamiento quizás haya que buscarla en una especie de escisión cartesiana que se ha hecho entre el "yo" materno como sujeto social, que parece que se va disolviendo durante la liturgia en paritorio, y el útero albergador de la gestación (Bodoque y Montes: 2003), sobre el que se centra la mayor parte de la atención. Como apunta Menéndez (2002), estaríamos ante una perspectiva biologicista de la atención hospitalaria que llevaría, en cierta medida, a cosificar a las mujeres en un abdomen -objeto sobre el que trabajar- invisibilizándolas y separándolas de la estructura social : «Yo le dije de oye por favor avisar a mi marido que llevo aquí cuatro horas que sepa cómo estoy, y nada, y nada y eso es lo peor (...) eso es lo 
peor, porque luego llaman y tampoco le dicen nada, no está bien, avisar a mi marido por favor y decirle que estoy bien, o si se puede subirlo, pues nada» (XXK). A tenor de los relatos, la atención de los profesionales parece estar focalizada en el control biométrico de los parámetros biológicos, desapareciendo la mujer tras el protagonismo de éstos (Hernández Garre y Echevarría 2015).

Los testimonios hablan, de esta manera, de un proceso asistencial impregnado de ideologías productivistas, en el sentido de que la lógica de trabajo se ensambla como una especie de cadena de producción, en la que, como diría Martin (1987), el personal sanitario ejerce de jefe que controla la elaboración, para que la mercancía sea socialmente viable: «Están haciendo pruebas pero nunca te dicen para qué son o qué es lo que te están haciendo, es todo muy mecánico (...) tú estás allí bueno y ahora qué, qué están haciendo» ( $x x \lambda$ ). Parece que lo más importante son los resultados obtenidos desde la tecnología. Como apuntan Hernández Garre y Echevarría (2014) estos determinan el buen funcionamiento del proceso, por ello desde perspectivas posthumanistas se da menor importancia a las relaciones no biométricas: «Están haciendo su trabajo, mirando el monitor y los resultados y tal, pero no les salía ni palabra» (xxi). Desde esta configuración laboral el acompañamiento se convierte en algo subsidiario, que se producirá sólo en función de las posibilidades que marque el proceso productor, e incluso, a veces, al margen de los deseos maternos: «Yo me hubiera gustado que hubiese estado allí, porque hombre el dolor no te lo va a quitar pero tienes una chispa de alivio, tú sabes dónde está él, él sabe dónde estás tú y quieras que no es otra cosa» (xxع). Se trata de una especie de exclusión estructural de los padres marcada por la mecanización asistencial y las rutinas institucionales: «A mí se me excluyó, se me excluyó de no decir oye suba usted al menos un ratito y esté con su mujer un rato, o tal, o cualquier cosa así, aparte no me tienen ni informado siquiera de decirme pues va bien la cosa (...) a mí me dejaron así como si no existiera por decirlo así, como si no existiera, como si no existiera» $(x y \varepsilon)$. Parece que la propia introversión del ceremonial dicta la clausura materna, quizás para ejemplificar la sacralidad clínica de lo que allí va a acontecer, como si la presencia paterna se relacionara con la profanación de los lugares consagrados a los nacimientos litúrgicos. La consecuencia es una diáspora de los padres, que ubicados como residentes de los 
«no lugares ${ }^{6}$ perciben la indiferencia de unos profesionales que a tenor de los testimonios se envisten en auténticos protagonistas del proceso (Hernández Garre y Echevarría, 2016): «Protagonista en lo que yo fui protagonista fue porque yo me impuse, y porque yo quise, y porque yo me metí por medio (...) ahí realmente protagonista, protagonista, en ese momento, cuando están todos los médicos, los únicos protagonistas que hay son los médicos» (xyס). Como diría Blázquez (2009), la propia dinámica laboral, mecanizada y productivista, no deja lugar a lo emotivo, lo importante es la biología, la ausencia de riesgo y los resultados epidemiológicos.

\section{Los habitantes de los «no lugares». El papel asignado a los padres durante el parto desde la perspectiva de género}

A las dificultades estructurales de esta especie de «régimen monacal» se suman además ciertos prejuicios profesionales, que catalogan a los padres como una especie de torpes rituales que aumentan el nerviosismo de las madres. Estaríamos, como apunta Brullet (1996), ante una repartición de tareas desde la que se atribuye a la mujer toda la responsabilidad, mientras al hombre se le desresponsabiliza y excluye ${ }^{7}$ : «Sí (...) porque como son listas ellas prefieren que no estén los hombres allí, y yo creo que no debería ser así, deberían más bien de incentivar al hombre, a decir bueno venga usted ayude aquí también, porque eso es normal, es algo normal que una persona como su marido vea lo que está pasando, sabes» ( $х у \mathrm{\kappa})$. Se reproduce así, de forma velada, la asignación de roles sexista que socialmente se otorga a hombres y mujeres en materia reproductiva (Maroto et al. 2009), perpetuándose los patrones tradicionales que implican la división sexual entre el trabajo reproductivo y el productivo. Parece que estuviéramos ante una especie de «sistema claustral», erigido en función de la comodidad de los sanitarios, en el que los padres fueran concebidos como fuera de sitio, como posibles fiscalizadores de sus actuaciones clínicas: «Imagino que cuando estás trabajando y tienes que hacer cosas que son delicadas, si delante tuya tienes una persona es más fácil hacerlo que si tienes dos, porque si hay más

\footnotetext{
${ }^{6}$ Se trata de una expresión acuñada por el antropólogo francés Marc Augé, para referirse a los lugares de transitoriedad que no tienen suficiente importancia para ser considerados como «lugares»

7 En este sentido, hay que apuntar que la distribución de tareas domésticas aún sigue siendo muy desigual, llevando las mujeres un mayor peso en cuanto a los cuidados parentales y las tareas domésticas (Brunet y Alarcon 2005; Bonaccorsi 1999).
} 
de una te pueden decir oye ¿qué estás haciendo?» ( $x y \delta)$. Fiscalización que no es concebida como tal por los padres, que opinan que los expertos no tienen porque sentirse examinados por su presencia: «Yo entorpecerle su trabajo no le iba a entorpecer en absoluto, ellos su trabajo yo solamente allí y ya está, (...) dando apoyo moral es lo único» $(x y \varepsilon)$. Da la impresión de estuviéramos ante dos universos conceptuales antagónicos, el de los profesionales, que encuentra en la reclusión litúrgica su «modus vivendi», y el de las madres y padres, que se rebela contra las causas ocultas de esta especie de retiro clínico.

A tenor de los relatos, parece pues que aún quedan muchas cuestiones por resolver en cuanto al papel de los padres en los partos, ya que, pese a las nuevas perspectivas con respecto a los roles de género, la participación paterna encuentra todavía muchas trabas. $Y$ todo ello, a pesar de que muchos padres expresan su predisposición por estar en los «escenarios quirúrgicos» donde se dirime la representación más importante de sus vidas: «Sí estar allí, cerca de ella (...) para estar a su lado así apoyándola, sí (...) porque he estado sufriendo afuera, y querer entrar $y$ detenerme porque no me dejaban» ( $x y \mathrm{k}$ ). En línea con lo apuntado por Gotzon Baraia (2001), se observa en las escenificaciones de la procreación, una actitud proactiva de muchos de los padres ${ }^{8}$, talante desde el que quieren colaborar en el ritual hasta sus últimas consecuencias: "A ser posible sí, siendo posible sí, para mí sí, yo no soy una persona que tenga miedo a la sangre o cosas así, yo me aguanto muy bien, yo sí me hubiera gustado estar presente, incluso hubiera bajado a quirófano para estar ahí» $(x y \varepsilon)$. Parece que los padres están dispuestos a remar contra viento y marea por muchos obstáculos que se les presenten en el camino. Los discursos hablan, pues, de un ritual realmente restrictivo, en el que las condiciones de clausura forman parte esencial de su naturaleza. Quizás por ello las prohibiciones no se produzcan sólo en cuanto al acceso al sistema, sino que una vez que la propia dinámica asistencial dicta que éste es posible continúan. El rol de los padres, en el «espacio sagrado» del paritorio, parece estar acotado por los expertos, de manera que les piden que cumplan las directrices y el papel asignado según las necesidades y la situación de cada momento. Su participación queda, así,

8 En este sentido hay que apuntar que también existen padres que no quieren estar presentes en el parto de sus hijos por razones de miedo, angustia o reparo, y otra parte, si bien están presentes, lo están con una actitud pasiva e incluso desinteresada, o simplemente por complacer a sus parejas, pero no por decisión propia. 
limitada a estar al lado de sus mujeres, mostrando calidez desde el lugar fijado por los profesionales. Las propias limitaciones orgánicas hacen que los hombres parezcan estar «fuera de lugar», y aunque están presentes siguen habitando el espacio de los «no lugares», parece como si su concurrencia se produjese de manera diferente, como un objeto dejado por alguien: «El personal, hombre excluirme no, pero tampoco, ni una cosa ni otra la verdad (...) en todo momento me quitaba para que pudieran pasar» $(x y \mu)$. En este sentido, parece que la forma en que los hombres son situados, y se sitúan, hace que su presencia no contribuya, per se, a reducir la atribución de roles reproductivos que a mujeres y hombres se les ha asignado históricamente. Por ello, no se sabe si su comparecencia conduce a la igualdad, o más bien refleja las desigualdades tradicionales. Distinciones que otorgan a las madres un rol de cuidadoras "innato", corolario de un supuesto «instinto maternal» fruto de su naturaleza femenina, y al hombre ciertos patrones de masculinidad que le empujan a negar lo relacionado con lo emocional en oposición a la feminidad (González de Chávez, 2001). En definitiva, como apunta Esteban (1998), parece que su presencia no siempre significa una mejora en cuanto a las desigualdades de sexo, sino que más bien sus modos de realización sirven para perpetuar los estereotipos sexuales relacionados con la procreación.

\section{Co-viviendo la maternidad. Tras el valor simbólico otorgado por madres y padres al acompañamiento durante el parto.}

A pesar de las dificultades estructurales para el acompañamiento, tanto las madres como los padres otorgan un alto valor simbólico al acompañamiento durante el parto. Los discursos de las madres están tamizados de satisfacción por la presencia de los padres en el proceso, desde su perspectiva su presencia los convierte en cómplices y colaboradores durante el trance: «Digo tú avísame cuando bajen las contracciones en la máquina porque el apoyo que me dice que baja, que baja (...) descansaba y decía ya bajan y me agradaba que estuviera ahí, luego que por ejemplo él me llevó un abanico y estaba abanicándome cuando me daba calor (...) pues también era un aspecto agradable en ese momento para mí, y si hubiera estado sola» $(x x \mu)$. Su presencia parece que las resignifica en el proceso, reconstituyendo ciertos roles sociales perdidos durante la dura liturgia clínica del paritorio. En esta misma línea, los padres que pudieron asistir muestran también un 
alto grado de satisfacción que opaca, en cierta medida, las marginaciones y restricciones vividas previamente: «Eso es una experiencia que no, que vamos que no tiene precio, ni con dinero ni con nada» (хуп); «Me he sentido muy de cerca de ella, y creo yo que todos los hombres deberían de pasar por ese momento para sentirlo y estar ahí con ellas porque es algo que conlleva un montón» (хук). Parece que como progenitores sienten la corresponsabilidad de la experiencia maternal, convirtiéndose, desde su óptica, en un elemento crucial que humaniza y devuelve en parte las dimensiones afectivas al proceso: «Esto es una cosa de los dos, aunque sea más de ella, pero que sí que es verdad, yo por lo menos intentaba tranquilizarla y animarla, sobre todo cuando tenía las contracciones, y motivarla y nada y quitarle un poco el miedo que tenía, yo sé que por mucho que le ayudara

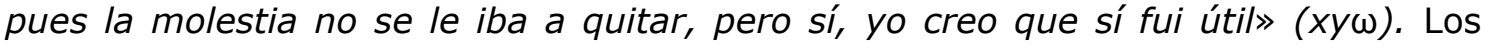
padres reclaman, de esta manera, un lugar ritual, sintiendo que cubren aspectos vitales de la sensibilidad de las madres que los expertos no pueden alcanzar, reintegrando y decosificando, en parte, a las madres en su condición de sujeto.: «Sí porque ya al estar con ella tenía un apoyo emocional, porque puede haber gente que estén más o menos cualificados, pero no son familiares, no es la persona a la que le está agarrando la mano» ( $x y \delta)$. Parece, pues, que la reclusión no es la mejor estrategia para cubrir las necesidades emocionales de las madres, y máxime cuando están en un lance que pone a prueba sus recursos más íntimos. Se trataría de una situación contradictoria en la que por un lado están las necesidades emocionales de las madres, que dictaminan en muchas ocasiones la pertinencia de un apoyo fraterno continuo, y por otro la propia dinámica asistencial mecanizada y la persistencia de ciertas ideologías de género, que dan prelación a las rutinas de exclusión.

\section{Conclusiones}

Los relatos hablan de un itinerario asistencial mecanizado, centrado en las rutinas clínicas, que lleva a las madres a una especie de reclusión ritual, y a los padres a la exclusión, o en el mejor de los casos a su ubicación como habitantes de los «no lugares». Configuración que las madres y los padres acatan a regañadientes, mientras luchan por recuperar un protagonismo, que según los testimonios parece que les han arrebatado los profesionales. Parece que las razones de la exclusión se encuentran en la propia configuración de un itinerario laboral productivista y taylorista, diseñado para la biointervención y la comodidad de los profesionales, 
posicionamiento desde el que los padres pueden ser percibidos como posibles fiscalizadores de las actuaciones clínicas. A ello se suma la persistencia de una serie de ideologías de género que atribuyen a las madres mayor responsabilidad en labores maternales, mientras a los hombres se les asignan ciertos patrones de masculinidad que los excluyen.

Pese a las dificultades, las madres y padres muestran su satisfacción cuando el acompañamiento fraterno pudo acontecer, considerando que la experiencia los convierte en cómplices y colaboradores del inicio de las vidas de sus bebés. En esta línea la mayoría de padres se muestran proactivos, reclamando un lugar ritual significativo durante el parto de sus parejas, ya que desde su perspectiva cubren aspectos vitales de la sensibilidad de las madres que los expertos no pueden alcanzar, descosificando y reintegrando a las madres, en cierta medida, en su condición de sujeto.

Parece que se trata, pues, de un tema cuya exégesis pasa por la compresión de la organización laboral en los centros clínicos, organización que da prelación a las rutinas biológicas quedando otros aspectos del proceso, como los emocionales o psicológicos, en un segundo plano. Disposición que de otra parte es muy consecuente con las ideologías productivistas y posthumanistas, que dan prelación a la biointervención sobre otras dimensiones del ser humano, y con ciertas perspectivas de género que construyen el ideario de la maternidad en oposición a los simbolismos atribuidos tradicionalmente a la masculinidad. El mantenimiento de esta estructura dependerá en parte, pues, de un cambio de ideologías que consiga introducir otras perspectivas más holísticas en los procesos clínicos de asistencia al parto hospitalario, sin por ello descuidar el control de riesgos, y de la asunción de roles de corresponsabilidad más igualitarios en tareas de maternidad. Seguramente desde estas cosmovisiones se enriquecerían las experiencias ligadas al acompañamiento durante los partos hospitalarios públicos, mostrando una mayor sensibilidad, no sólo formal y legislativa, sino efectiva, durante las rutinas clínicas. Configuraciones desde las que se pudieran cubrir ciertas necesidades emocionales que la rigidez de los procesos asistenciales actuales no parecen contemplar en su plenitud. 


\section{Bibliografía}

American College of Nurse-Midwives. (2013). El dolor durante el trabajo de parto. Journal of Midwifery \& Women's Health, Volume 58, Issue 2: 243-244. DOI: 10.1111/jmwh.12002

Baroni, F.; Vilela L. y Scorsolini F. (2013). Significados da gravidez e da maternidade: discursos de primíparas e multíparas. Psicología: teoría e práctica, 15(1): 19-32. Disponible en: Disponible en: <http://pepsic.bvsalud.org/scielo.php?script=sci_arttext\&pid=S1516$36872013000100002 \&$ Ing=pt\&nrm=iso >. Fecha de acceso 20 mayo 2016.

Blázquez, M.L. (2009). Ideologías y prácticas de género en la atención sanitaria del embarazo, parto y puerperio: el caso del área 12 de la Comunidad de Madrid. Tesis doctoral, Universidad d Rovira i Virgili, Tarragona.

Bertaux, D. y Bertaux, I. (1993). Historias de vida del oficio de panadero. En J.M Marinas y C. Santamaría (eds.), La historia oral: Métodos y experiencias. Madrid: Debate, 231-250.

Bodoque, Y. y Montes, Ma.J. (2003). El cuerpo del embarazo: poder, discursos y representaciones, en Actas del IX Congreso de Antropología FAAEE. Simposio 6. Cultura, poder y salud. Barcelona: soporte CD-ROM.

Bourdieu, P., \& Wacquant, L.J.D. (1995). Respuestas. Por una antropología reflexiva. México: Grijalbo.

Bonaccorsi, N. (1999). El trabajo femenino en su doble dimensión: doméstico y asalariado. La Alijaba, Revista de Estudios de la mujer, 4, 83-92.

Brullet, C. (1996). Prácticas de crianza e identidades parentales. En P. Carrasquer y C. Brullet, coord., Sociología de las relaciones de género. Madrid: Instituto de la Mujer, Ministerio de Asuntos Sociales, 45-65.

Brunet, I., \& Alarcón, A. (2005). Mercado de trabajo y familia. Revista de Investigaciones Políticas y Sociológicas, 4 (2), 115-129.

Caprara, A. \& Pandim, L. (2008). Ethnography: its uses, potentials and limits within health research, Interface-Comunicação, Saúde, Educação, 12(25): 363-376. DOI: http://dx.doi.org/10.1590/S1414-32832008000200011 
Coffey A., \& Atkinson P. (2005). Encontrar el sentido a los datos cualitativos. Medellín: Universidad de Antioquia de Medellín.

Esteban, M.L. (1998). ¿Y los hombres?, Inguruak-Revista Vasca de Sociología y Ciencia Política, 22 : 15-29. ISSN: 02147912.

Enkin, M., Lumley, J. \& Keirse, M. J. (1995). Social and professional support during childbirth. En: Effective care in pregnancy and childbirth. New York. Oxford University Press.

Enkin, M., Keirse, M.J.N.C.,\& Renfrew, M. (1989). A guide to effective care in pregnancy and childbirth. Oxford: Oxford University Press.

Federación de Asociaciones de Matronas de España, FAME. (2008). Documento de iniciativa a favor del parto normal. Madrid.

González de Chávez, A. (2001). Preparación para el parto versus preparación para la maternidad y paternidad. En Género y salud. I Jornadas de la Red de Médicas y Profesionales de la Salud. Madrid: Instituto de la mujer, 24-31.

Gotzon Baraia, J. (2001). Convivencia y reestructuración de los roles. En P. Pérez Fuentes, el., Modelos y referentes de los comportamientos masculinos y femeninos en la juventud vasca. Vitoria-Gasteiz: EmaKunde, Instituto Vasco de la mujer.

Grele, R.J. (1991). La historia y sus lenguajes en la entrevista oral, quién contesta a las preguntas de quién y por qué. Historia y Fuente oral, 5: 111-129. Disponible en: <http://www.jstor.org/stable/27753314>. Fecha de acceso 5 mayo 2016.

Hemminki, E.; Virta, A.L. y Koponen, P. (1990). A trial on continuous human support during labour: feasibility, interventions and mother's satisfaction. Journal of psychosomatic Obstetrics and Gynecology; 11(2), 239-250. DOI: http://dx.doi.org/10.3109/01674829009084420.

Hernández Garre, J.M. (2013). La labor del antropólogo. Reflexiones epistemológicas. Murcia: Diego Marín.

Hernández Garre, J.M, \& Echevarría, P. (2014). La parte negada del parto institucionalizado. Explorando sus bases antropológicas, Revista de 
Dialectología y Tradiciones Populares, 69: 2, 327-348. DOI: http://dx.doi.org/10.3989/rdtp.2014.02.004.

Hernández Garre, J.M, \& Echevarría, P. (2015). El nacimiento hospitalario e intervencionista. Un rito de paso hacia la maternidad, AIBR, Revista Iberoamericana de Antropología, 10:3, 401-426. DOI: http://dx.doi.org/10.11156/aibr.100306.

Hernández Garre, J.M, \& Echevarría, P. (2016). Sociología del parto. Conflictos asistenciales en el marco del paradigma tecnocrático, Revista Internacional de Sociología, 74:1, DOI: http://dx.doi.org/10.3989/ris.2016.74.1.025.

Hodnett, ED., Gates, S., \& Hofmeyr, GJ. (2012). Continuous support for women during childbirth. Cochrane Review, In: Cochran Database of Systematic Reviews;Oxford: Update Software. DOI: 10.1002/14651858.CD003766.pub4.

Hodnett, ED., Gates, S., Hofmeyr, GJ., \& Sakala, C. (2013). Continuous support for women during childbirth. Cochrane Review, In: Cochran Database of Systematic Reviews;Oxford: Update Software. DOI: 10.1002/14651858.CD003766.pub5.

Hofmeryr, G.J., Nikodem, V.C., Wolman, W.L,; Chalmers, B.E., \& Kramer, T. (1991). Companionship to modify the clinical birth environment: effects on progress and perception of labour and breast feeding. Journal Obstetric $\begin{array}{llll}\text { Gynecological. } & 98 & \text { (2), } & \text { 756-64. }\end{array}$ http://dx.doi.org/10.4102/curationis.v21i1.596.

Ibañez, J. (1979). Interpretación y análisis del discurso. En I. Jesús en Más allá de la sociología. El grupo de discusión, técnica y crítica, Madrid: Siglo XXI, 333351.

Janes, C.R., Stall, R., \& Gifford, S.M. (1986). Anthropology and Epidemiology. Dordrecht, Netherlands: Reidel Publishing Company.

Klaus, M.H., \& Kennel, J.H. (1993). Pais e bebes: a formação do apego. Porto Alegre, Artes Médicas.

Kleinman, A. (1988). The illness narratives. Suffering, healing and the human condition. New York: Basic Books. 
Kleinman, A. \& Benson, P. (2004). La vida moral de los que sufren enfermedad y el fracaso existencial de la medicina. En Monografías Humanitas no 2, Dolor y Sufrimiento en la práctica clínica, Barcelona: Fundación Médica y Humanidades Médicas, 17-26.

Lally, J.E., Murtagh, M.J., Macphail, S., \& Thomson, R. (2008). More in hope than expectation: a systematic review of women's expectations and experience of pain relief in labour. BBC Medicina, 6(7). DOI: 10.1186/1741-7015-6-7.

Ley 3/2009 de 11 de Mayo de Derechos y Deberes de los usuarios del Sistema Sanitario Público.

Luque, M. A., \& Oliver, M.I. (2005). Diferencias culturales en la percepción y vivencia del parto. El caso de las mujeres inmigradas. Index Enfermería, 14: 48-49.DOI: http://dx.doi.org/10.4321/s1132-12962005000100002.

Maroto, G., Castaño, E., García, M.M., Hidalgo, N., \& Mateo, I. (2009). Paternidad y servicios de salud. Estudio cualitativo de las experiencias y expectativas de los hombres hacia la atención sanitaria del embarazo, parto y posparto de sus parejas. Rev. Esp. Salud Pública, 83 (2): 267-278. DOI: http://dx.doi.org/10.1590/s1135-57272009000200010.

Martin, E. (1987). The Woman in the Body. Boston: Beacon Press.

Mauss, M. (1966). Sociologie et anthropologie. París: PUF.

Menéndez, E.L. (2000). Factores culturales. De las definiciones a los usos específicos. En E. Perdiguero y J.M. Comelles, (eds.), Medicina y Cultura. Estudios entre la Antropología y la Medicina. Barcelona: Bellaterra, 163-188.

Menéndez, E.L. (2002). La parte negada de la cultura. Barcelona: Ediciones Bellaterra.

Ministerio De Sanidad y Consumo, MSC. (2007). Estrategia para la atención al parto normal. Madrid: Autor.

Ministerio de Sanidad y Política Social, MSPS. (2010a). Guía de Práctica Clínica sobre la atención al parto normal. Madrid: Autor.

Ministerio de Sanidad y Política Social, MSPS. (2010b). Estrategia Nacional de Salud Sexual y Reproductiva. Madrid: Autor. 
Organización Mundial de la Salud, OMS. (1985). Declaración de Fortaleza. Tecnología apropiada para el parto. Ginebra: Autor.

Organización Mundial de la Salud, OMS. (1996). Cuidados en el parto normal: una guía práctica. Ginebra: Autor.

Romaní, O. (1992). Marginación y drogodependencias. Reflexiones en torno a un caso de investigación. En Álvarez Uría (Comp.), Marginación e inserción. Los nuevos retos de las políticas sociales. Madrid: Eudymión, 259-281.

Sociedad Española de Obstetricia y Ginecología, SEGO. (2008). Recomendaciones sobre la asistencia al parto normal. Madrid: Autor.

Schwarz, Patricia. (2008). Las lesbianas frente al dilema de la maternidad. En M. Pecheny, C. Figari \& D. Jones (Coord.), Todo sexo es político. Buenos Aires: Del Zorzal, 193-214.

Taylor, S.J. \& Bogdan, R. (1987). Introducción a los métodos cualitativos de investigación: la búsqueda de significados. Barcelona: Editorial Paidós.

Van Gennep, A. (1986). Los ritos de paso. Estudio sistemático de las ceremonias de puerta y del umbral. Madrid: Taurus. 